تعريف ووصف لذباب الفاكهة المصاحب لعوائل مختلفة من أشجار الفاكهة في بعض بساتين طرابلس

$$
\begin{aligned}
& \text { إيمان الطاهر الزنتاني } \\
& \text { جامعة طرابلس، كلية الزراعة، قسم وقاية النبات، ليييا. } \\
& \text { emanzntn@yahoo.co.uk }
\end{aligned}
$$

https://doi.org/10.36602/jmuas.2019.v01.01.27

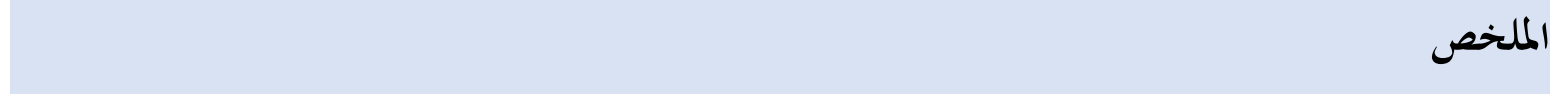
استهدفت هذه الدراسة الحقلية التعرف على أنواع ذباب الفاكهة التي تصيب ثمار الفاكهة ببعض مناطق طرابلس خلال الموسمين الزراعيين 2016 2017، بإستخدام طريقة التربية في الثمار Fruit rearing ، وشملت الدراسة عوائل: الجوافة Psidium guajava L.) Guava)

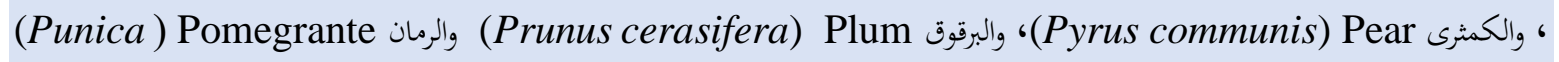
،(Prunus persica) Peach والخوخ ،granatum أظهرت النتائج وجود ثلاثة أنواع من ذباب الفاكهة التابع لفصيلة Tephritidae، اثنان منها تنتمي للجنس Bactrocera وهما: ذبابة ثمار الخوخ Bactrocera zonata (Saunders) تصيب جميع العوائل، وذبابة . Bactrocera sp على عائل الجوافة بمنطقة عرادة، وكذلك ذبابة فاكهة البحر الأبيض المتوسط Ceratitis capitata (wiedemaan) سجل وجود ذبابة التين الأفريقية Zaprionus indianus (Gupta) التابعة لفصيلة Drosophilidae لأول مرة في ليبيا على عائلي الرمان والجوافة.

كلمات مفتاحية: Tephritidae- طرابل-Taprionus indianus - Bactrocera

المقدمة

تتغذى العديد من الآفات الحشرية على الثمار في بساتين الفاكهة، متسببة في إحداث خسائر اقتصادية هامة، خاصة الأنواع التي تتبع رتبة ذات الجناحين (Order: Diptera)، وفصيلة ذباب الفاكهة الحقيقي ( Family: Tephritidae)، التي تضم حوالي 500 جنس و4000 نوع، تتغذى حوالي 40\% منها على الثمار (White and Elson-Harris, 1994)، وتتبع

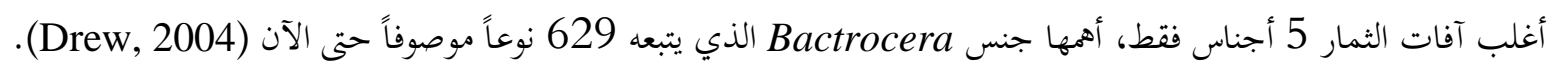

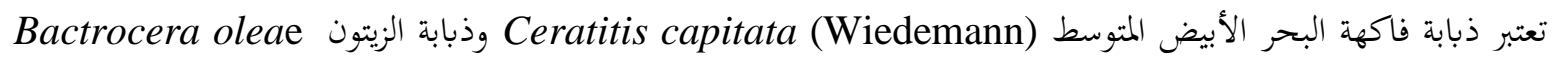
Damiano, 1961; De Cillis, ) من الآفات الإقتصادية الهامة في يبيا وقد سجل وجودهما منذ سنة 1920م (Gmelin) 338 
1920)، في السنوات القريبة الماضية أشارت نشرة إرشادية إلى وجود ذبابة الحوخ B. zonata (Saunders) لأول مرة في ليبيا (الزناتي، 2010)، وهي آفة منشؤها جنوب وجنوب شرق قارة آسيا (White and Elson-Harris, 1994)، وصنفت على أها تمثل تحديدا خطرا لإنتاج الفاكهة في دول الشرق الأوسط وشمال أفريقيا وبدرجة أقل في جنوب أوربا (EPPO, 2008).

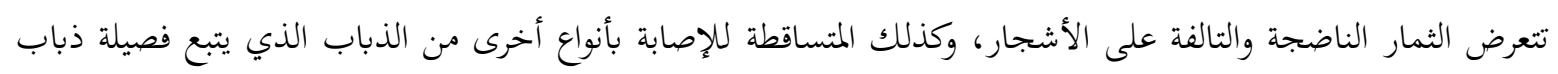
الفاكهة (Family: Drosophilidae) وفصيلة (Family: Lonchaeidae) وهي غالباً آفات ثانوية تضع بيضها داخل الثقوب التي سببتها أنواع من فصيلة ذباب الفاكهة (Family: Tephritidae)، بينما تضع بعض وانواع ذباع ذباب فصيلة بيضها في الثمار التالفة. (Family: Muscidae)

دراسة هذه الآفات بغرض التعرف عليها، توصيفها أو المكافحة يتم بجمع ذباب الفاكهة بعدة طرق من ضمنها طريقة التربية في الثمار fruit rearing method والتي تعتبر فعالة في حالة أنواع فصيلة Tephritidae لأها عادة تقضي معظم دورة حياها داخل الثمار المصابة على الأشجار، وكذلك تستخدم طرق أخرى كالمصائد التي هما طعوم البروتين المتحلل تستخدم كجاذبات لذباب الفاكهة من فصائل وأجناس خختلفة (Navarro-Llopis, et al., 2008)، ويستخدم الجاذب الغذائي methyl eugenol أيضاً لجذب ذكور بعض أنواع BPPO/OEPP, 2005) Bactrocera). استهدفت هذه الدراسة تعريف ووصف أنواع ذباب الفاكهة التي جمعت من ثمار مصابة لبعض أنواع أشجار الفاكهة في بعض بساتين طرابلس.

\section{المواد وطرق البحث}

1. منطقة الدراسة: بمدينة طرابلس وبالتحديد: طريق المطار (جنوب طرابل)، ومحطة أبحاث كلية الزراعة بنطقة سيدي

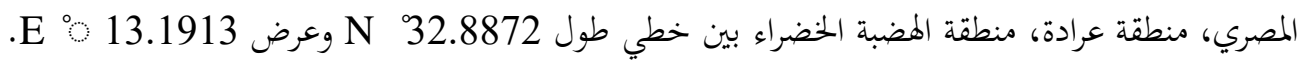
2. العوائل النباتية: الجوافة Psidium guajava L. Guava والكمثرى Pyrus communis Pear والبرقوق Plum Prunus persica Peach والحون Punica granatum Pomegrante والرمان Prunus cerasifera

جمعت عدد 25 ثرة من كل عائل من العوائل المختارة بشكل عشوائي من البساتين في صيف 2016 و2017 ونقلت إلى

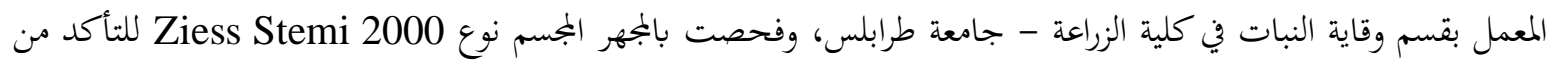
وجود مظاهر إصابة بذباب الفاكهة، اتبعت طريقة التربية في الثمار fruit rearing method للحصول على البالغات بغرض 339 
التعرف عليها، حيث وضعت كل ثمرة داخل حافظة بلاستيكية شفافة، مجهزة سلفاً بوضع منديل ورقي ملفوف بقاعدةا للسماح للطور اليرقي الأخير بالتعذر، وقفلت الحافظات بأغطية مثبت عليها قطع شاش حتى تسمح بالتهوية (شكل، 1). تركت العينات في ظروف المعمل عند درجة حرارة 25 × 2 مم، ورطوبة نسبية 65\% × 20، وفحصت يومياً حتى ظهور بالغات الذباب التي حفظت في الكحول الإيثيلي بتركيز 70\%، لغرض الفحص والتعريف من خلال خصائصها الظاهرية وباستخدام المفاتيح التقسيمية، وكررت العملية ثلاث مرات لكل عائل نباتي.

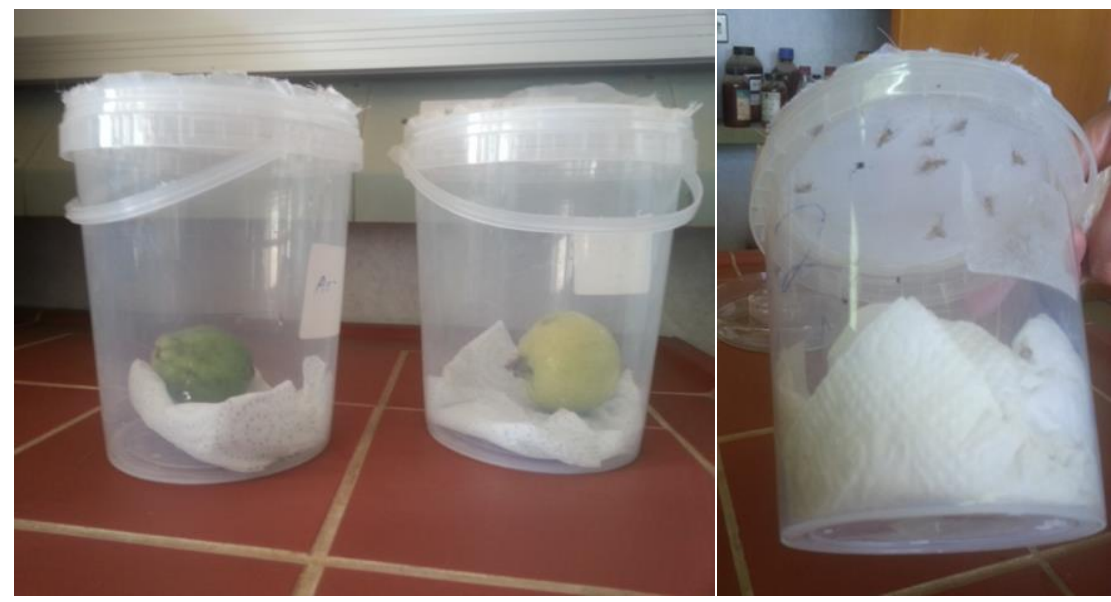

شكل (1) طريقة تربية ذباب الفاكهة في ثمار العوائل المختارة

\section{النتائج والمناقشة}

أظهرت النتائج وجود نوعين من ذباب الفاكهة ينتميان للجنس Bactrocera ونوع ثالث ينتمي للجنس Ceratitis، وتتبع الأنواع الثلاثة فصيلة ذباب الفاكهة الحقيقي (Family: Tephritidae) وذلك لوجود التصاميم المميزة في الأجنحة، وكذلك تميزها بانثناء عرق الجناح تحت الضلعي Sc إلى أعلى بشكل زاوية قائمة تقريباً 90ه، ثم يخف مظهره إلى ثنية وهذه من أهم الخصائص المميزة للفصيلة (EPPO/OEPP, 2005)، وسجل وجود نوع رابع ينتمي للجنس Zaprionus التابع لفصيلة ذباب الفاكهة (Yassin and David, 2010) (Fam: Drosophilidae). من خلال الفحص المجهري للخصائص المورفولوجية للعينات المجمعة، وبالإستعانة ببعض المفاتيح التقسيمية لذباب الفاكهة (Plant Health Australia, 2016; Prabhakar, et al., 2012)، تم تصنيف ووصف الأنواع الآتية: 


\section{1: ذابة الحوخ Bactrocera zonata (Saunders)}

يتميز الرأس بوجود عيون مركبة كبيرة يخرج من بينها في منطقة التجويف الجبهي (antennal fossa) قرن الاستشعار الأريستي،

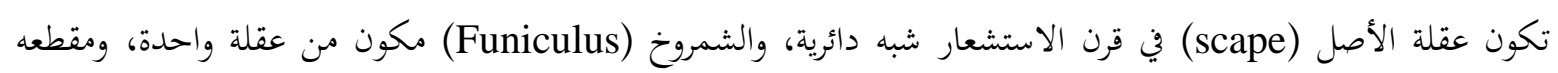
العرضي مثلث عريض وله سطحين داخلي وخارجي، يخرج من السطح الخارجي أريستا طويلة قوية متماثلة في الجنسين.

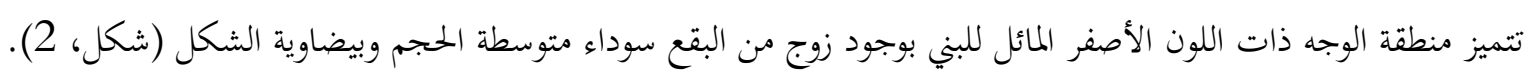

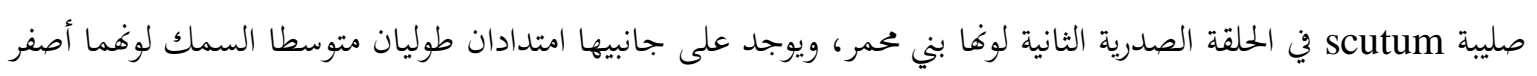

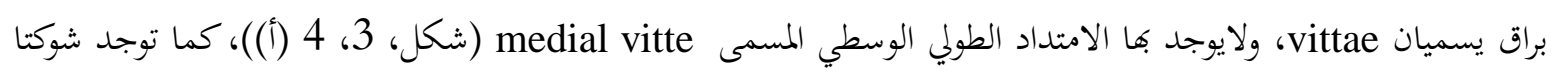
anterior supra-alar setae prescutellar setae

تتميز الفصوص notopleura postpronotal بلوغا الأصفر (شكل، 3 و4 (ب))، كذلك تكون صليبة Scutellum صفراء اللون أيضاً (شكل،4،3)، ولاتوجد شوكتان في قاعدةا، ولكن تتميز بوجود شوكتين قميتين (شكل، 2 و3).

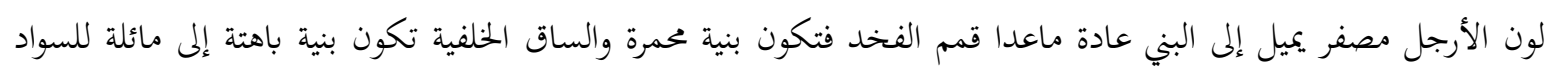

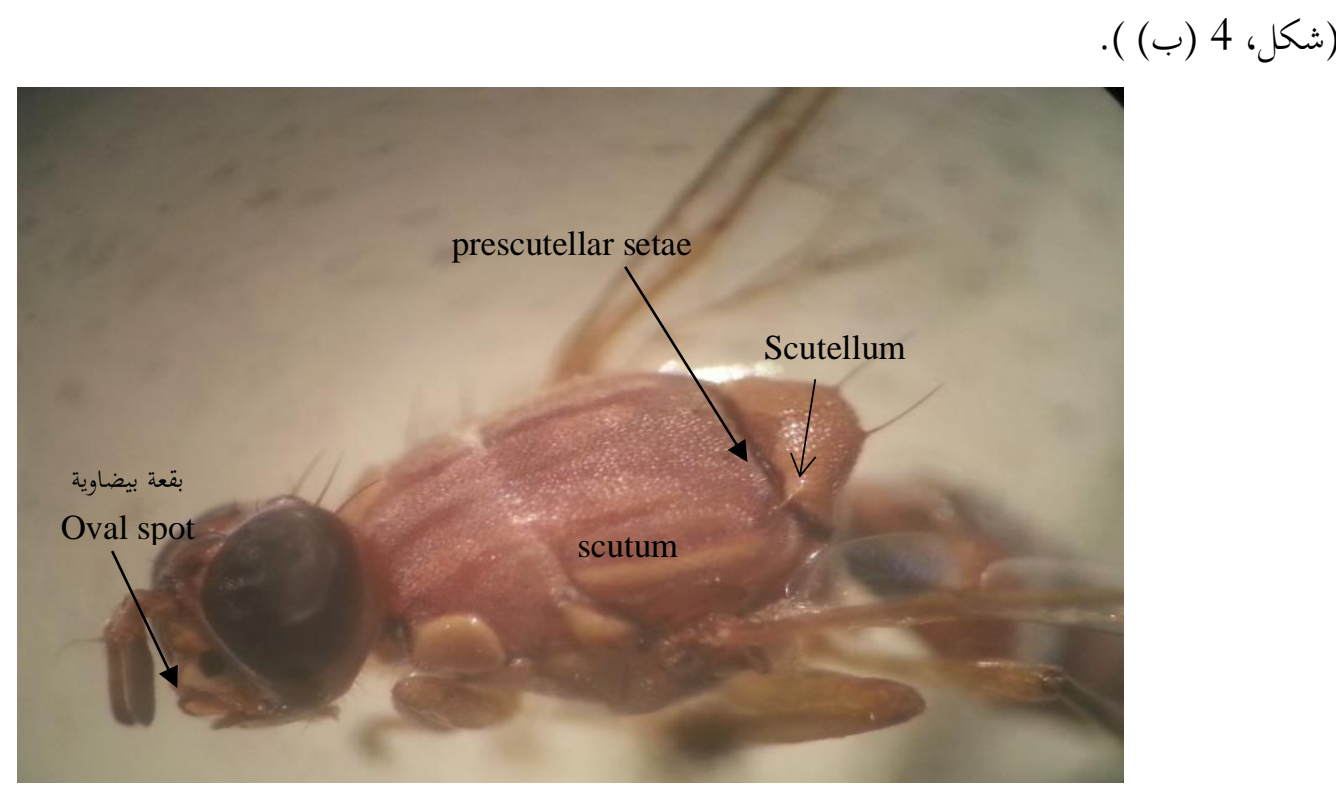

B. zonata (Saunders) شكل (2). منظر جانبي لذبابة الحوخ 


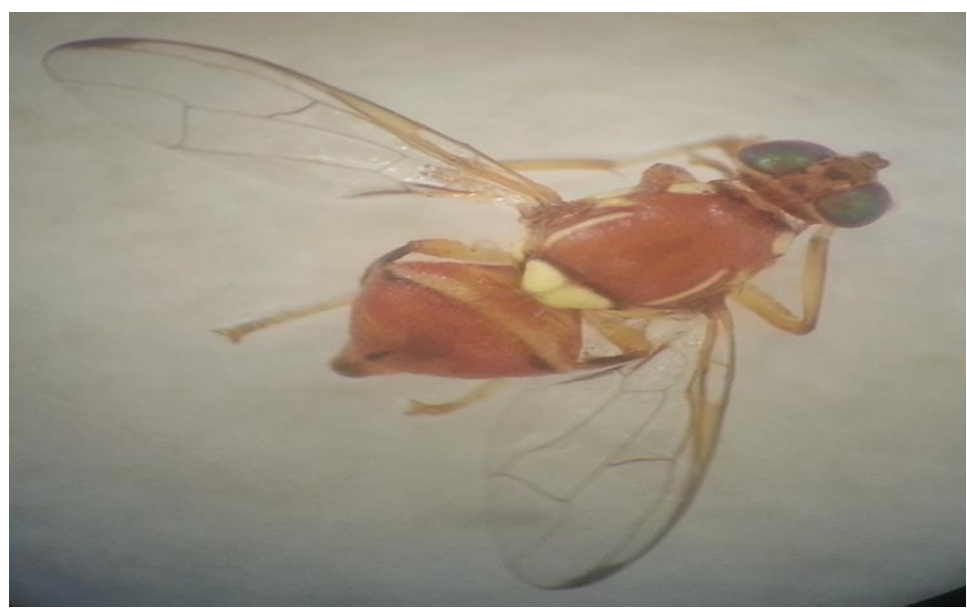

B. zonata شكل (3). الأنثى البالفة لذبابة الحوخ

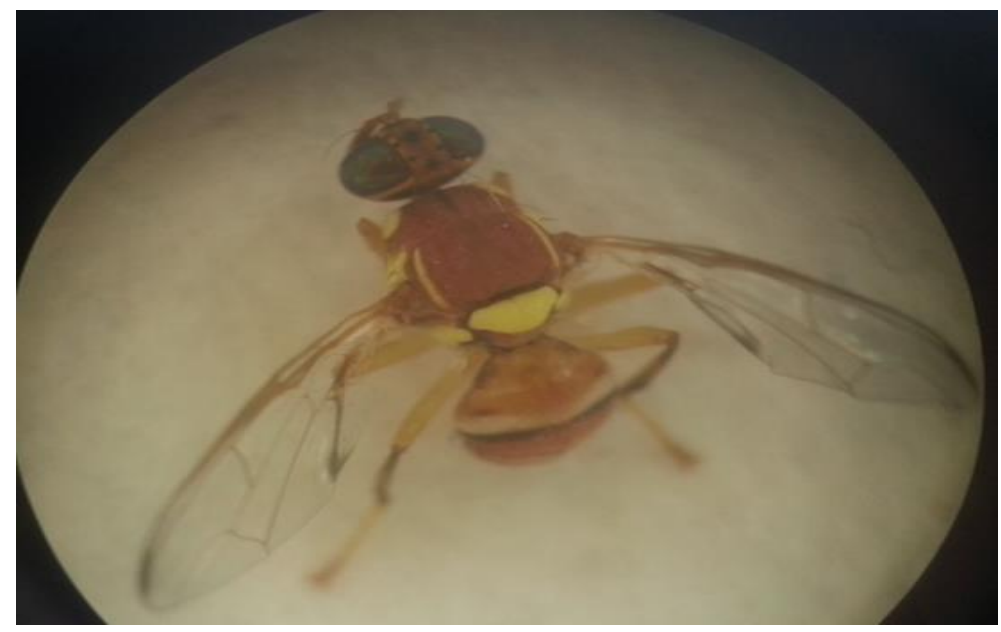

شكل (4، أ). منظر ظهري لذكر ذبابة الحوخ Bonata من البرقوق

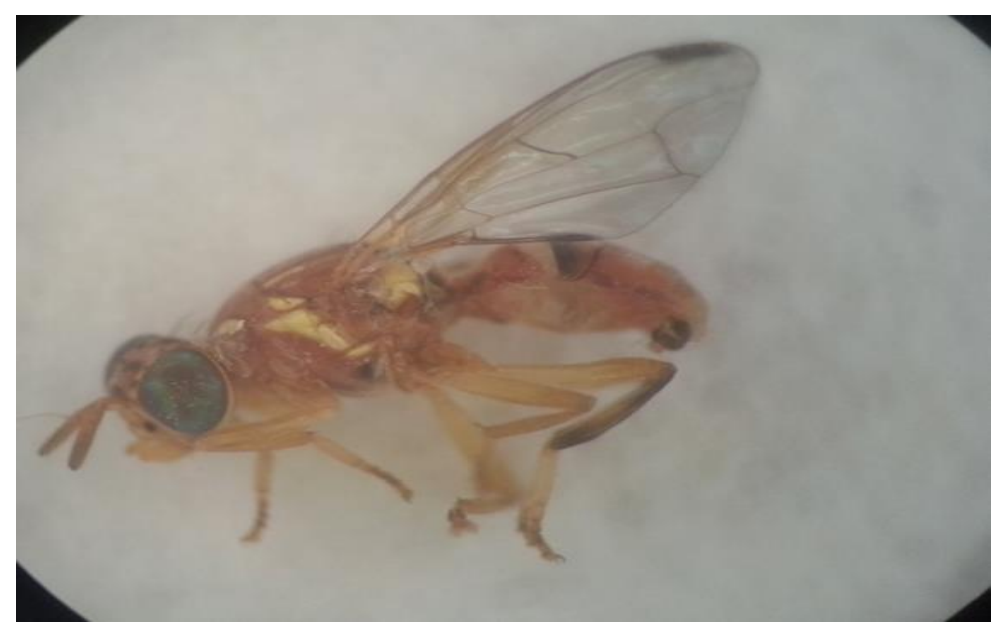

B. zonata 
يتميز الجناح الغشائي بغياب الشعرة الحسية Microtrichiae في المنطقة القاعدية للخلية br، ويكون العرق الضلعي Costal مein vنتلاً أو يستمر بشكل ضيق جداً إلى فاية العرق R2+3 قبل أن يمتد إلى بقعة في قمة الجناح (شكل، 5أ، 5ب ). يكون البطن بيضاوي متطاول، وتلتحم فيه ترجتا الحلقتين الأولى والثانية، بينما تكون ترجات الحلقات البطنية الأخرى غير ملتحمة، بحيث تكون حوافها الجانبية غير مستوية، تتلون ترجتا الحلقات الثالثة والرابعة باللون البني المحمر، مع وجود حزام ضيق

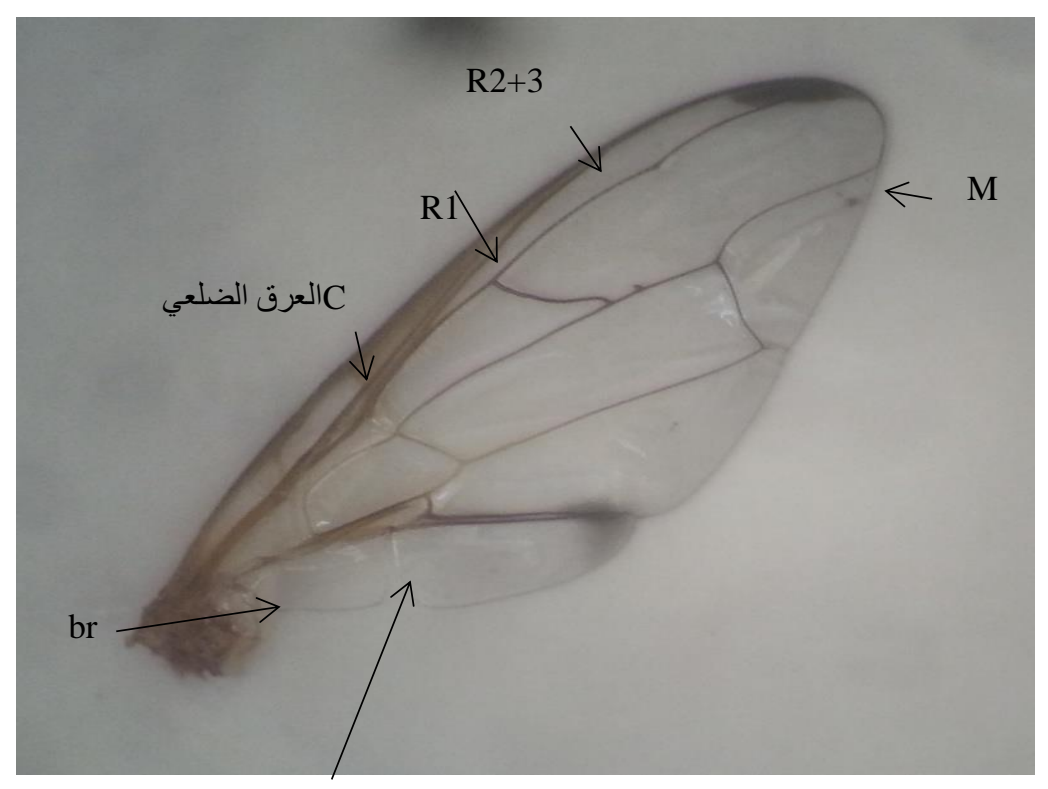

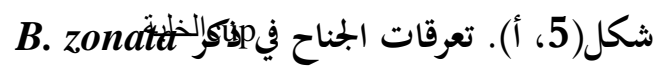

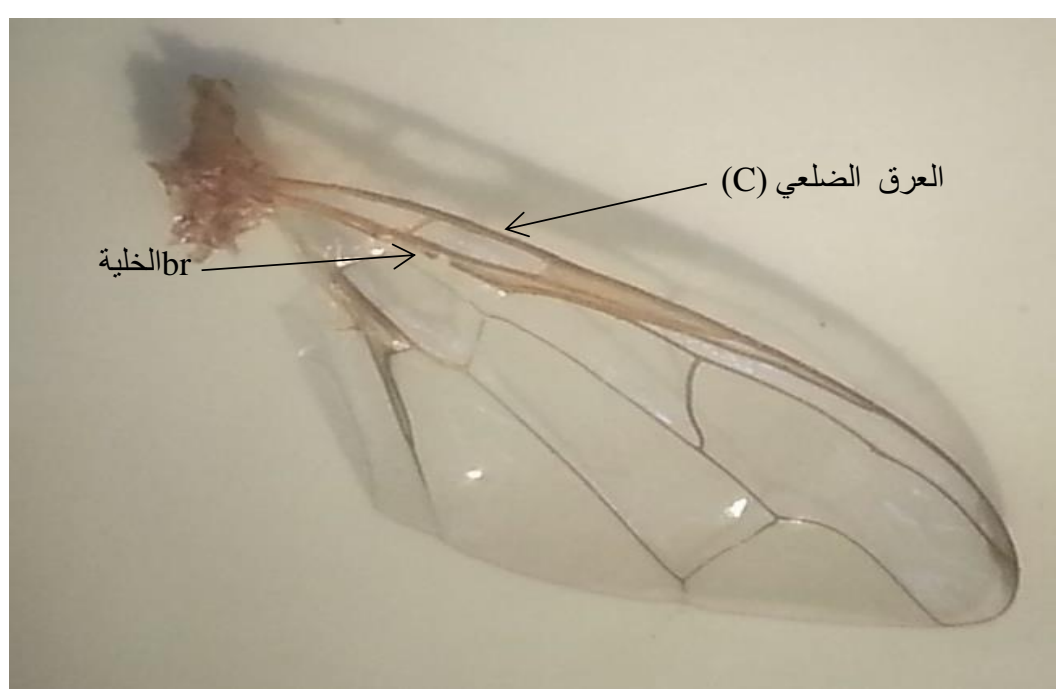

Bكل(5) ب) B. Bonata تعرقات الجناح في أنثى 
قاتم اللون متد على طول الحافة الأمامية للترجة البطنية الثالثة، هذا الحزام القاتم عادة غير متصل في المنطقة الوسطى منه، بالإضافة إلى وجود خط طولي أسود متمد على أجزاء من منتصف الترجة البطنية الرابعة والخامسة (شكل، 3و6)، كما توجد زوايا سوداء جانبية ضيقة على الحلقتين الرابعة والخامسة، وزوج من البقع بيضاوية بنية محمرة ولامعة على ترجة الحلقة الرابعة، تتميز آلة وضع البيض بنهايتها الصغيرة الإبرية الحادة (aculeus) (شكل، 3)، ويتميز الفص الخلفي لزوج ملحقات ترجة العقلة البطنية التاسعة (شكل، 6) في الذكر بقصره (شكل، 4ب)، وبوجود شعيرات على جانبي البطن تسمى (surstylus)

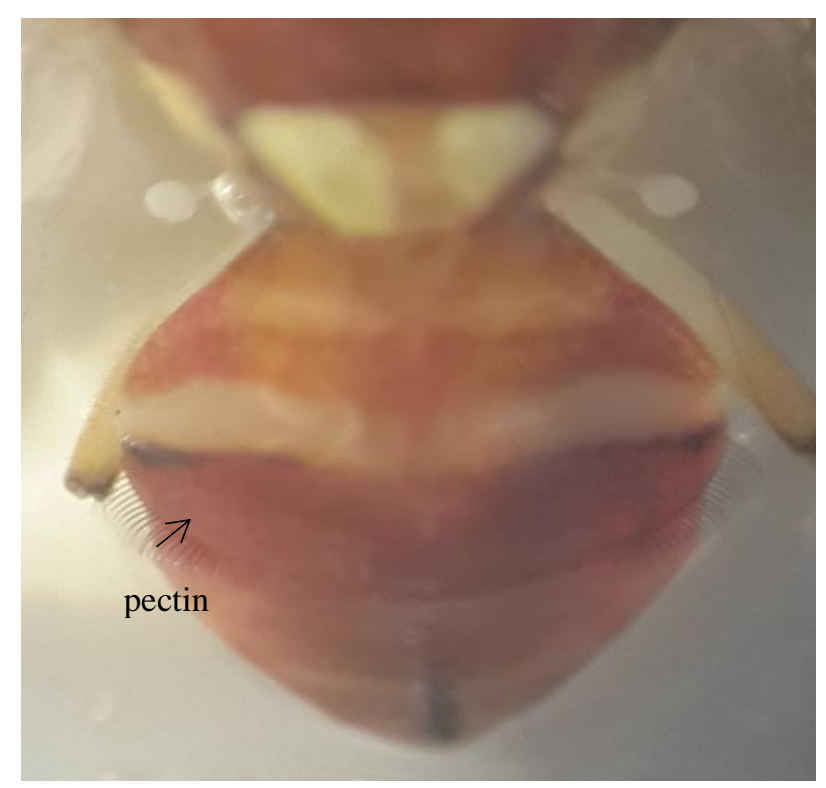

شكل (6) منطقة البطن في ذكر B. zonata وتظهر على الجانبين شعيرات pectin

:Bactrocera sp. ذبابة الفاكهة

يتميز الرأس بوجود عيون مركبة كبيرة يخرج من بينها في منطقة التجويف الجبهي (antennal fossa) قرن الاستشعار الأريستي. تكون عقلة الأصل (scape) في قرن الاستشعار شبه دائرية، والشمروخ (Funiculus) غير مقسم ومقطعه العرضي مثلث عريض وله سطحين داخلي وخارجي، يخرج من السطح الخارجي أريستا طويلة قوية متماثلة في الجنسين. تتميز منطقة الوجه ذات اللون الأصفر المائل للبني بوجود زوج من البقع سوداء متوسطة الحجم وبيضاوية الشكل (شكل، 7). 


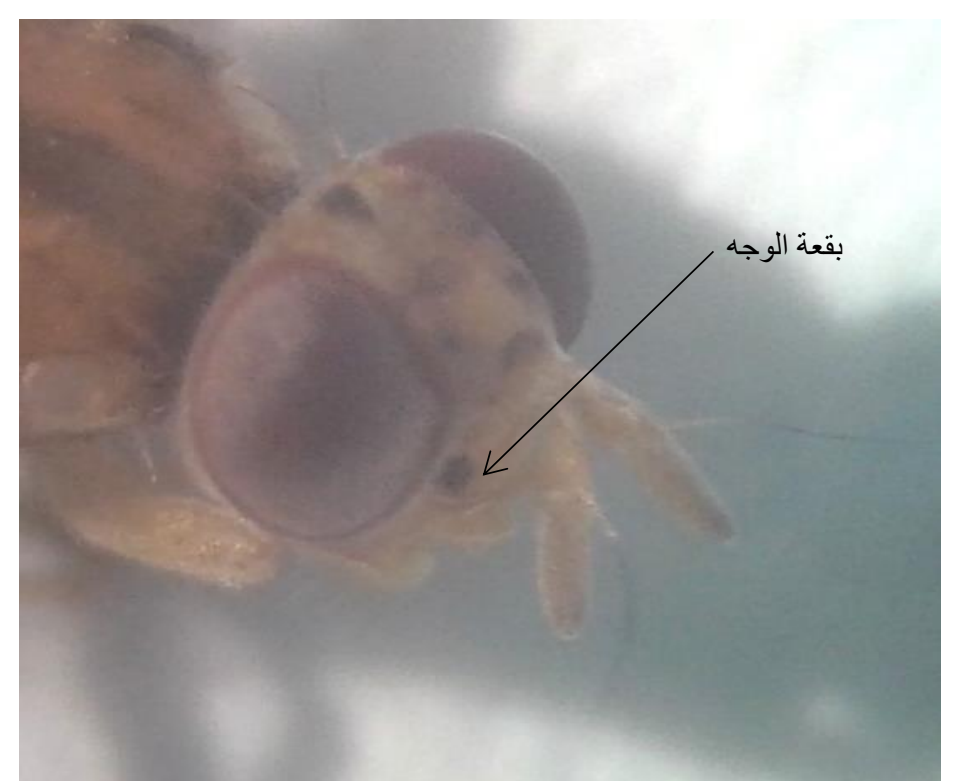

شكل (7). منطقة الرأس لذبابة الفاكهة . Bactrocera sp على عائل الجوافة

تتميز الصليبة الظهرية Scutum في الحلقة الصدرية الثانية بكوها بنية محمرة، ولكن يتخللها مساحات تميل إلى السواد في منتصفها كما يوجد امتدادان طوليين متوسطي السمك لوغما أصفر براق يسميان vittae على جانبيها محاطان بشريطين أسودي اللون (شكل، 8 أ، 8 ب). البطن بيضاوي ومتطاول، تلتحم فيه ترجتا الحلقتين الأولى والثانية فقط، أما بقية ترجات البطن فهي منفصلة عن بعضها وبالتالي حوافها الجانبية غير مستوية، يوجد شريطان باهتا اللون ممتدان كحزام على ترجة الحلقتين البطنية الأولى والثانية، كما يوجد حزام أسود اللون عريض ومتصل بالكامل ممتد على طول الحافة الأمامية للترجة البطنية الثالثة، في منتصفه يتقاطع مع خط طولي أسود ممتد على طول الترجة البطنية الثالثة والرابعة والخامسة بحيث يشكل علامة حرف T (شكل،8أ، 8 ب)

. (Prabhakar,et al. 2012) 


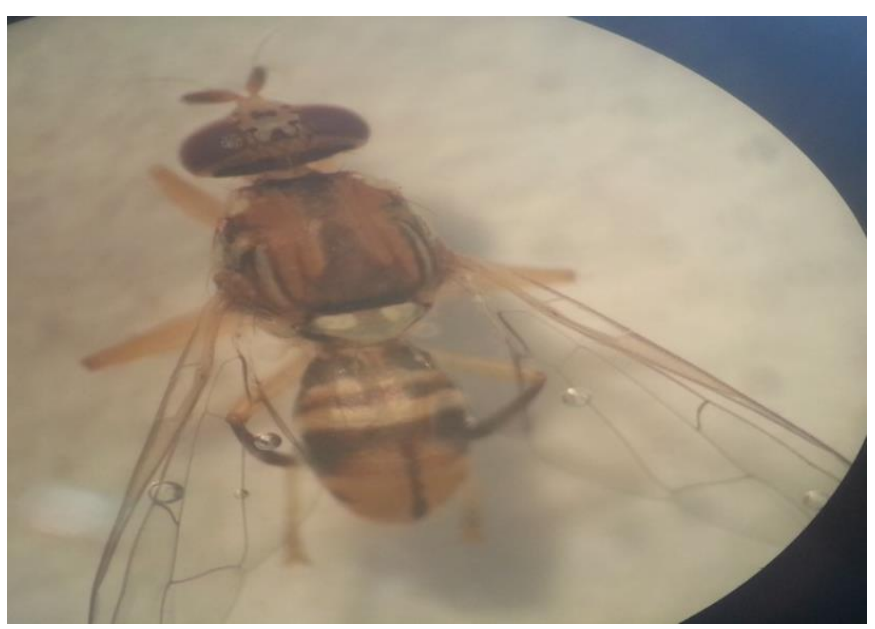

شكل (8 8 أ). منظر ظهري لذبابة الفاكهة . Bactrocera sp على عائل الجوافة

3. ذابة البحر الأبيض المتوسط Ceratitis capitata (Wiedemann)

يكون الرأس كبير الحجم، وقرن الاستشعار أريستي، يتميز الذكر عن الأنثى بوجود زائدتين ماسيتي الشكل لوفما أسود ممتدتان على قمة شوكتي orbital الأماميتين (شكل، 9).

تتميز صليبة الحلقة الصدرية الثانية scutum بلوغالأصفر مع وجود مساحات كبيرة سوداء اللون بتصميم ميز، تكون صليبة

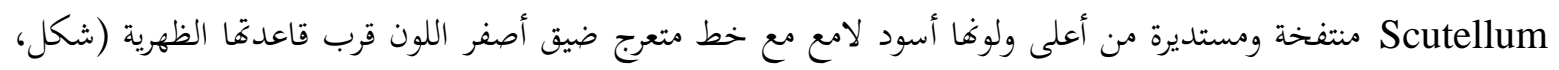

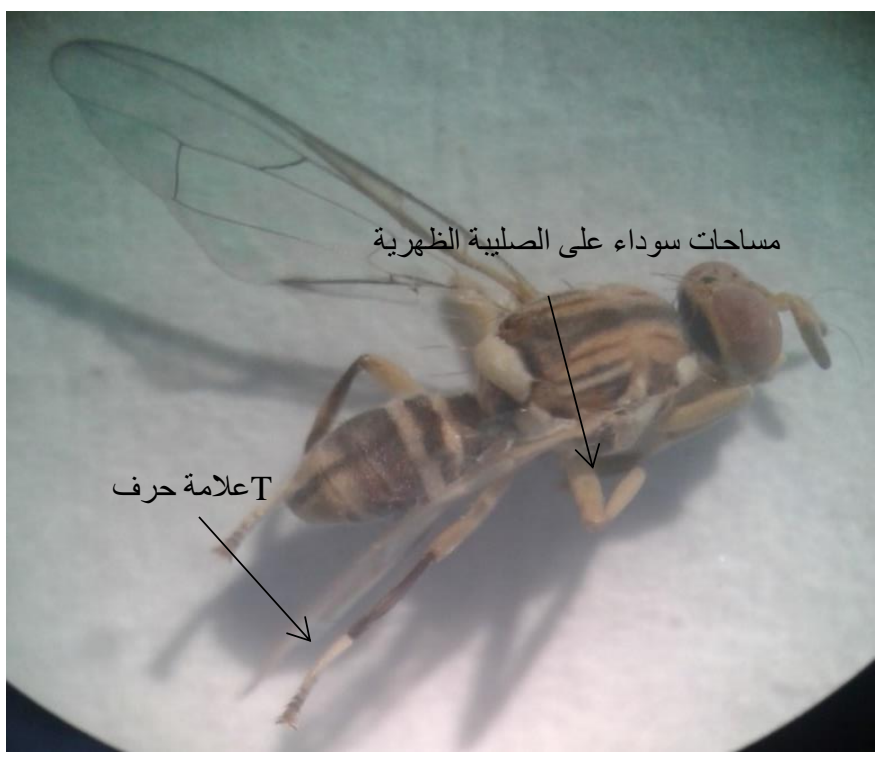

شكل (8 ب). منظر جانبي لذبابة الفاكهة . Bactrocera sp على عائل الجوافة 


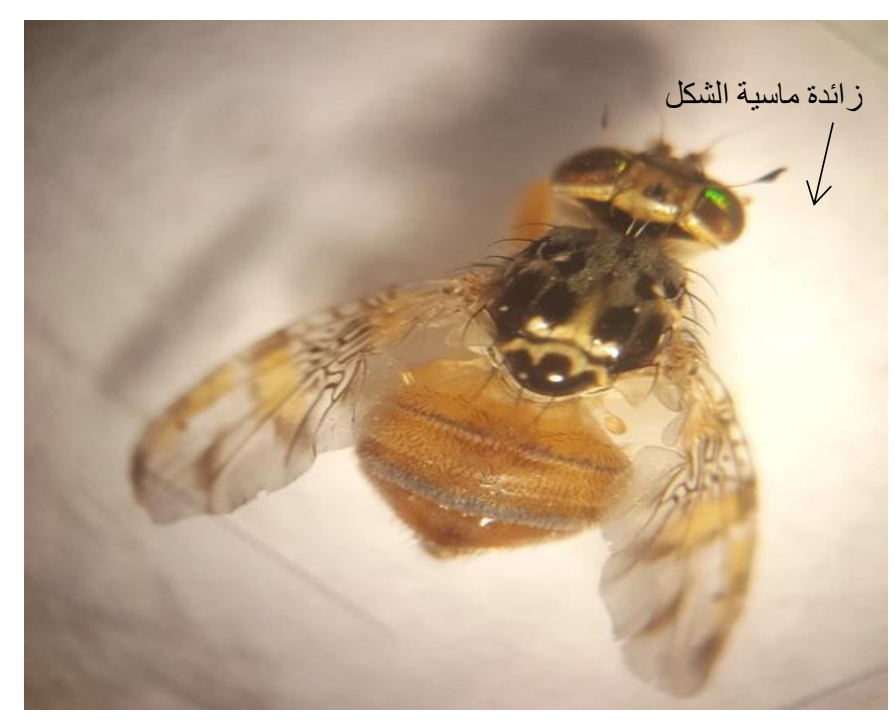

شكل (9). ذكر (C. capitata (Wiedemann) على الجوافة بمنطقة طريق المطار

الجناح عريض بالنسبة لطوله ولونه أصفر معتم مع ثلاث أشرطة أو أحزمة بنية في ثلثي مساحته الطرفية، وهي منفصلة عن بعض، ويوجد في خلايا نصف الجناح تقريبا عديد من الخطوط دقيقة وصغيرة وغير منتظمة وداكنة اللون (شكل، 10).

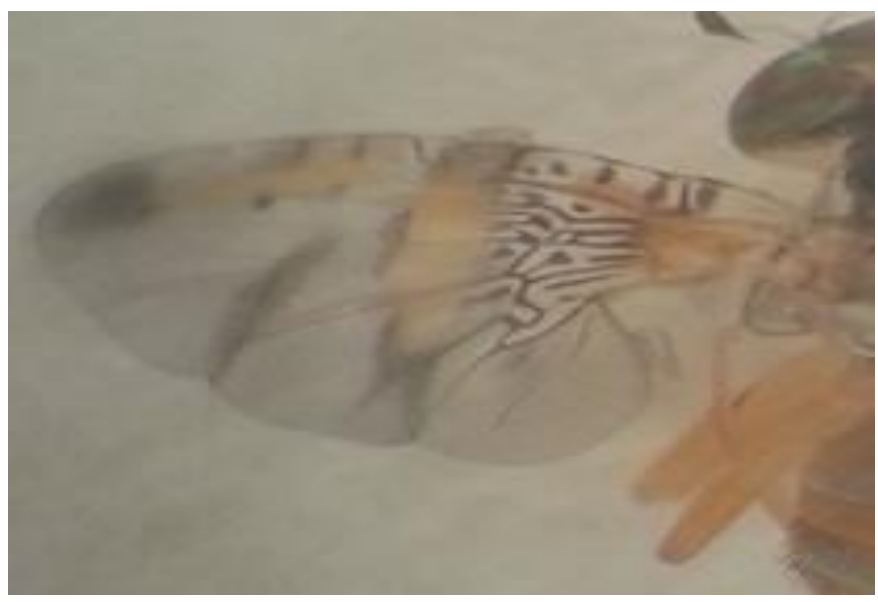

C. capitata (Wiedemann) شكل (10). الجناح في ذكر

البطن مصفر اللون ويوجد على ترجات الحلقات البطنية شريطان مستعرضان لوغما بني باهت (شكل،9)، يبرز من موخرة بطن الأنثى آلة وضع البيض (شكل، 11). 


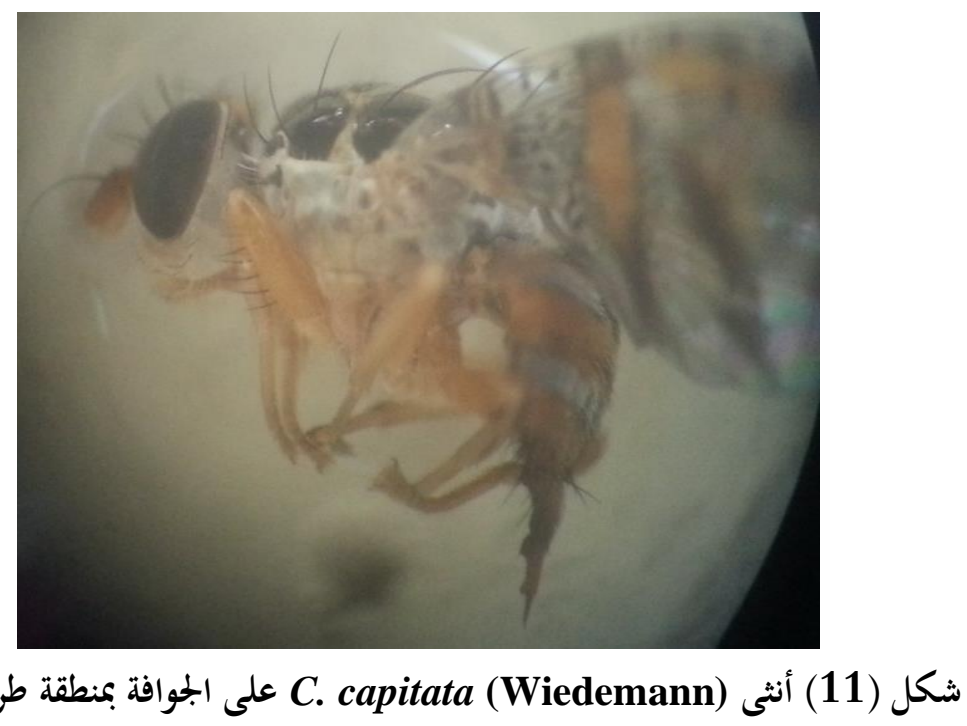

شكل (11) أنثى C. capitata (Wiedemann) على الجوافة بمنطقة طريق المطار

4aprionus indianus (Gupta 1970) ذبابة التين الأفريقية

(Family: تم تحميع العديد من البالغات من الثمار المصابة، وهي تتبع للجنس Zaprionus (Frosophilidae) التي تتميز بصغر أحجامها مقارنة بأنواع ذباب الفاكهة التابعة لفصيلة (Family: Tephritidae) ومن خلال الفحص المجهري للخصائص المورفولوجية للعينات المجمعة، وبالاستعانة ببعض المفاتيح التقسيمية لذباب الفاكهة (Yassin and David, 2010) )، تم تعريف النوع بناء على الخصائص التصنيفية التالية: يتميز الرأس بوجود عيون مركبة كبيرة يخرج من بينها في منطقة التجويف الجبهي (antennal fossa) قرن الاستشعار الأريستي، وتتميز الأريستا بوجود شعيرات طويلة تعطيها مظهرا ريشيا (شكل، 12)

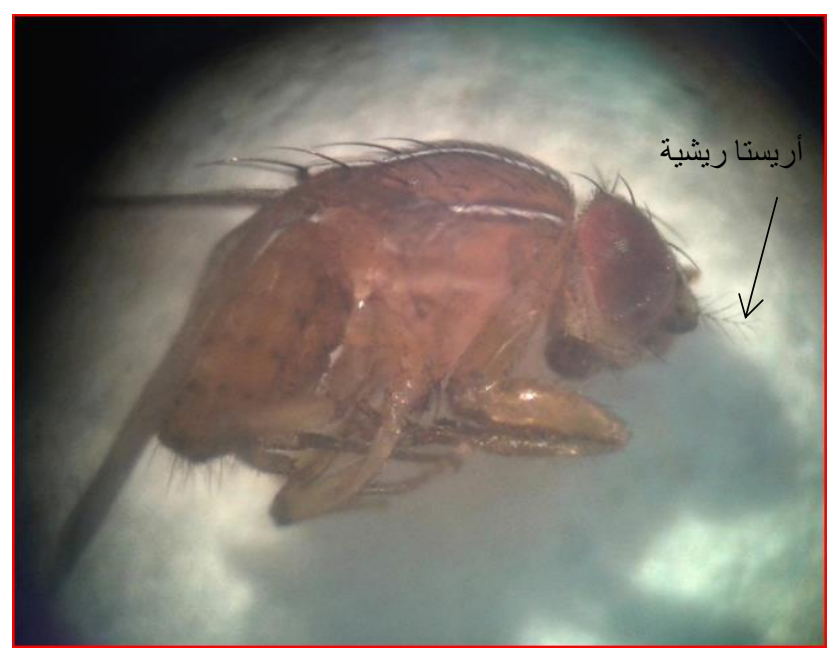

شكل (12) ). منظر جانبي لذبابة التين الأفريقية Z. indianus .معت من الرمان 
الرأس والصدر لوغما بني عحمر، توجد على منطقة الرأس والصدر أشرطة طولية سوداء (black vittae) محيطة بأشرطة طولية بيضاء اللون (white vittae) ومتثدة في ابتاه الخلف، وتتميز بأن عرضها ثابت، لايوجد على الصدر أشرطة طولية تحت وسطية (شكل،12ب).

يوجد في منطقة الفخذ للأرجل الأمامية صف من الأشواك ملتحمة عند قاعدةا مع شعرات طويلة قوية وسميكة (bristles)

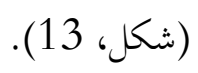

البطن لوها مصفر فاتح، يتميز غطاء العضو الذكري Aedeagal flap بكونه أملساً من القمة ولكن مسنن قاعدياً، وتتميز الة وضع البيض oviscape بوجود ستة شعيرات حسية (Peg-like ovisensilla). من الحقائق الهامة أن كثافة التبادل التجاري للمواد الزراعية كالفواكه والخضروات عالمياً كانت له تأثيراته السلبية على الإنتاج

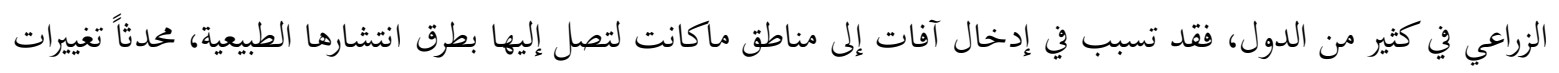
كبيرة في ديناميكيات العشائر والعلاقات المتبادلة داخل الأنظمة البيئية (De Meyer, et al., 2010) -

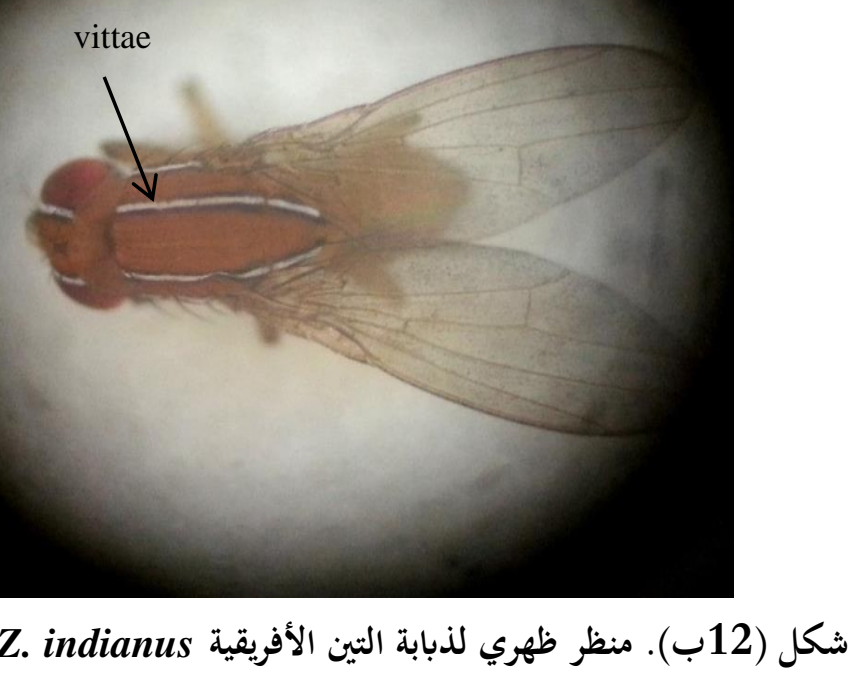

شكل (12ب). منظر ظهري لذبابة التين الأفريقية Z. indianus جمعت من الحوخ 


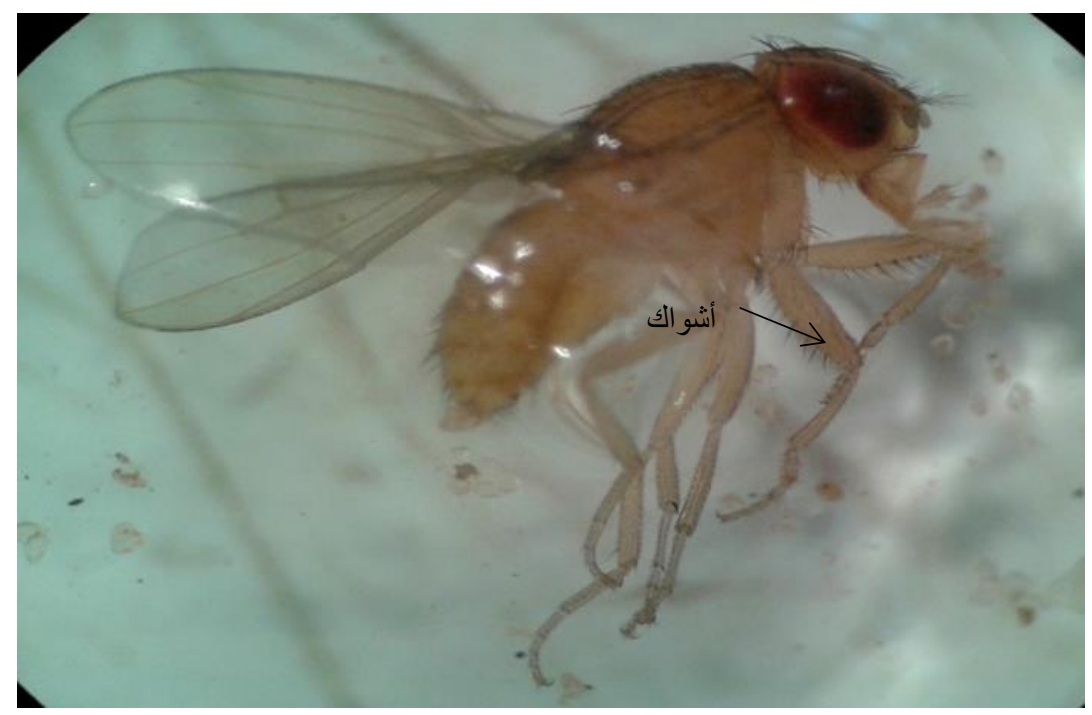

من عائل الرمان (Gupta 1970) - Indianus (Gu) شكل (13).

ومن بين أهم هذه الآفات، أنواع ذباب الفاكهة من فصيلة (Fam: Tephritidae)، والتي بالرغم من أن كثيرا من أنواعها C. capitata المهمة اقتصاديا منشؤها أفريقيا ومنها انتشرت إلى أنحاء متفرقة من العالم، كذبابة فاكهة البحر الأبيض المتوسط (De Meyer, et al., 2002) ) إلا أن كثافة التبادل التجاري جعل أفريقيا عرضة للعديد من أنواع ذباب الفاكهة الأخرى Drew, (الذي منشؤه جنوب Dactrocera التابع ل مجنوب شرق أسيا، ويضم أكثر من 629 نوعاً 2004)، في ليبيا وحتى وقت قريب، كان النوع B. oleae وهو المتخصص على ثمار الزيتون هو النوع الوحيد التابع لهذا الجنس والمسجل منذ بدايات القرن الماضي (De Cillis, 1920)، إلا أنه ونتيجة لكثافة استيراد الفواكه من الدول المجاورة وضعف جهاز الحجر الزراعي الخارجي في منافذ الدخول، ذكر وجود ذبابة الخوخ B. zonata (Saunders) لأول مرة خلال العقد الأول من القرن الواحد والعشرين (الزناتي، 2010)، ومن خلال النتائج المتحصل عليها في هذه الدراسة الحقلية تبين أن أغلب أعداد ذباب الفاكهة الذي تم الحصول عليه من ثمار الفاكهة المصابة هو من نوع ذبابة الخوخ B. Zonata (Saunders) جميع المواقع التي ثملتها الدراسة وعلى جميع العوائل المختبرة، وبالرغم من أن ذبابة الخوخ قد دخلت حديثا، مقارنة بذبابة فاكهة البحر الأبيض المتوسط De Cillis, 1920) C. capitata) إلا أنه من خلال هذه الدراسة لوحظ انتشارها السريع وأثبتت أها منافس قوي لها، حيث سجل وجودها على ثمار البرقوق والرمان والخوخ والجوافة في محطة أبحاث كلية الزراعة بمنطقة سيدي المصري، كما سجل وجودها على الجوافة والكمثرى في منطقة طريق المطار جنوب طرابلس، وعلى عائل الجوافة في منطقة الهضبة الخضراء ومنطقة عرادة. 
بالإضافة إلى ذبابة الخوخ أثبتت النتائج وجود نوع آخر من جنس Bactrocera, سجل لأول مرة على الجوافا بمنطقة عرادة وهو يشترك في عدة خصائص مع ذبابة الحوخ إلا أنه أصغر قليلا في الحجم مع وجود اختلافات في تلون منطقة الصدر حيث توجد مساحات سوداء أو تميل إلى السواد في منطقة الصليبة الظهرية Scutum ووجود حزام أسود سميك يمتد على الحافة الأمامية للحلقة البطنية الثالثة، يتقاطع في منتصفه مع شريط طولي أسود ممتد في منتصف ترجات الحلقات البطنية مكونا شكل حرف T واضحاً Prabhakar,et al. (2012) بالرغم من أن ذبابة التين الأفريقية Z. indianus منشؤها الأصلي أفريقيا والشرق الأوسط إلا أخا سجلت حديثاً نسبياً في مناطق عديدة من الشرق الأوسط كالأردن والسعودية ومصر(Al-jboory and Katbeh-Bader, 2012) ، وفي أماكن أخرى من العالم كالولايات المتحدة (Van Der Linde et al., 2006; Joshi et al., 2014)، سجلت هذه الآفة الثانوية في هذه الدراسة لأول مرة في ليبيا في سنة 2016 على عائل الرمان في مطة أبحاث الكلية، كما سجلت على الجوافة من منطقة عرادة، وعلى كل من عائل الجوافة والكمثرى من منطقة طريق المطار.

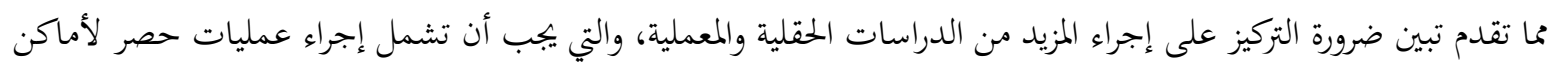

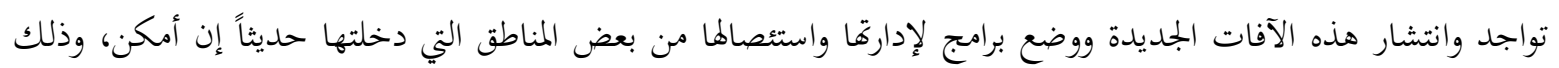

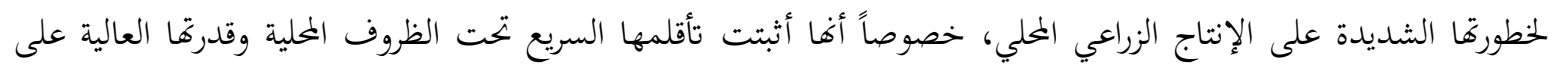
المنافسة والمدى العوائلي الواسع، الأمر الذي يتطلب خطوات حاسمة في سبيل عرقلة عملية انتشارها إلى مناطق جديدة بالبلد، كذلك إرشاد وتوجيه المزارعين للاهتمام باتباع إجراءات المكافحة الزراعية مثل جمع الثمار المتساقطة أو التي لازالت على الأشجار بعد انتهاء الموسم باعتبارها أساسية لتقليل الكثافة الإبتدائية لعشائر ذباب الفاكهة في البساتين، كذلك -وهو على قائمة الأولويات- يجب تفعيل الحجر الزراعي الدولي والداخلي لمنع دخول آفات جديدة أو إنتقال الآفات بين المناطق, فالحجر الزراعي يعتبر خط الدفاع الأول ضد دخول آفات جديدة للبلد.

\section{المراجع}

الزنائ، عادل. أ. م. (2010). دراسة موسمية وكفاءة المصايد الجاذبة لذبابة ثمار الحوخ بضواحي طرابلس - ليبيا. رسالة ماجستير. قسم علم الحيوان، كلية العلوم. جامعة الفاتح. مان.

Al-jboory, I., and A. Katbeh-Bader. (2012). First record of Zaprionus indianus (Gupta, 1970) (Drosophilidae: Diptera) in Jordan. World Applied Sciences Journal, 19(3): p. 413-417.

Damiano, A. (1961). Elenco delle specie di insetti dannosi ricordati per La Libia fino al 1960. Amministrazione Della Tripolitania. p 81.

\section{1}


De Cillis, E. (1920). Cinque anni di sperimentazione agrarian in Tripolitania. L' Agricoltura Coloniale. Vol. XIV, n. 1. E seguenti.

De Meyer, M., R. S. Copeland, R. A. Wharton, and B. A. McPheron. (2002). On the geographic origin of the Medfly Ceratitis capitata (Wiedemann) (Diptera:Tephritidae proceedings of $6^{\text {th }}$ International Fruit Fly Symposium 6-10 May 2002. Stellenbosch, South Africa, p. 45-53.

De Meyer, M., S. Ekesi, M. Virgilio, F. Khamis, M. Mwatawala, and A. Malacrida. (2010). In and out of Africa: invasion history of fruit flies on the African. $8^{\text {th }}$ international symposium on fruit flies of economic importance. Valencia, Spain.

Drew, R.A.I. (2004). Biogeography and speciation in the Dacini (Diptera: Tephritidae: Dacinae). Bishop Museum Bulletin in Entomology, 12: p. 165-178.

EPPO/OEPP.( 2005). Bactrocera zonata. OEPP/EPPO. Bull., 35: p. 371- 373.

EPPO. (2008). Bactrocera zontata., Available at:

http://www.eppo.org/Quarantine/bactrocera_zonata/bactrocera.htm.

Joshi, N. K., D. J. Biddinger, K. Demchak, and A. Deppen. (2014). First report of Zaprionus indianus (Diptera: Drosophilidae), in commercial fruits and vegetables in Pennsylvania. J. Insect. Sci., 14(259): p. 1-4.

Navarro-Llopis, V., F. Alfaro, J. Dominguez, J. Sanchis, and J. Primo. (2008). Evaluation of traps and lures for mass trapping of Mediterranean Fruit Fly in citrus Groves. Journal of Economic Entomology, 101(1): p. 126-131.

Plant Health Australia. (2016). The Australian handbook for identification of fruit flies. Version 2.1. Plant Health Australia. Canberra, ACT.

Prabhakar, C. S., P. Sood, and P. K. Mehta. (2012). Pictorial keys for predominant Bactrocera and Dacus fruit flies (Diptera: Tephritidae) of north western Himalaya. Arthropods, 1(3): p. 101-111.

Van Der Linde, K., G. J. Steck, K. Hibbard, J. S. Birdsley, L. M. Alonso, and D. Houle. (2006). First records of Zaprionus indianus (Diptera: Drosophilidae), a pest species on commercial fruits from Panama and the United States of America. Florida Entomologists, 89(3): p. 402- 404.

White, I. M., and M. Elson-Harris. (1994). Fruit Flies of Economic Significance: Their Identification and Bionomics. CAB International, Wallingford, Oxon, UK. 601 pp.

Yassin, A., and J. R. David. (2010). Revision of the Afrotropical species of Zaprionus (Diptera: Drosophilidae), with descriptions of two new species and notes on internal reproductive structures and immature stages. ZooKeys, 51: p. 33-72. 


\title{
Identification and description of Fruit fly species associated with different hosts in some Tripoli Fruit Orchards
}

\author{
Eman A. Zentani \\ Department of Plant Protection, \\ Faculty of Agriculture, \\ University of Tripoli \\ emanzntn@yahoo.co.uk
}

\section{https://doi.org/10.36602/jmuas.2019.v01.01.27}

\begin{abstract}
The present field study was carried out to identify fruit fly species in Tripoli fruit orchards during $2016-2017$ seasons by using fruit rearing method.

Five hosts were examined in this study namely Guava Psidium guajava L. , Pear Pyrus communis،, Plum Prunus cerasifera, pomegranate Punica granatum and Peach Prunus persica

The results revealed that three species of fruit flies that belong to family Tephritidae were identified; the Peach fruit fly, Bactrocera zontana (Saunders) and the Mediterranean fruit fly Ceratitis capitata (Wiedemaan), which were found in all fruit trees examined, and Bactrocera sp.that was recorded for the first time on Guava in the area. The fourth fruit fly species recorded was the African fig fly, Zaprionus indianus (Gupta) in pomegranate and Guava fruits.
\end{abstract}

